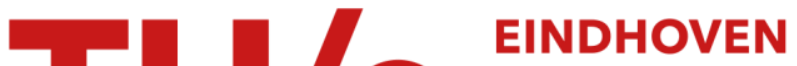 UNIVERSITY OF TECHNOLOGY
}

\section{Regularization of moving boundaries in a Laplacian field by a mixed dirichlet-neumann boundary condition: Exact results}

\section{Citation for published version (APA):}

Meulenbroek, B., Ebert, U., \& Schäfer, L. (2005). Regularization of moving boundaries in a Laplacian field by a mixed dirichlet-neumann boundary condition: Exact results. Physical Review Letters, 95(19), 195004-1/4.

[195004]. https://doi.org/10.1103/PhysRevLett.95.195004

DOI:

10.1103/PhysRevLett.95.195004

Document status and date:

Published: 01/01/2005

\section{Document Version:}

Publisher's PDF, also known as Version of Record (includes final page, issue and volume numbers)

\section{Please check the document version of this publication:}

- A submitted manuscript is the version of the article upon submission and before peer-review. There can be important differences between the submitted version and the official published version of record. People interested in the research are advised to contact the author for the final version of the publication, or visit the $\mathrm{DOI}$ to the publisher's website.

- The final author version and the galley proof are versions of the publication after peer review.

- The final published version features the final layout of the paper including the volume, issue and page numbers.

Link to publication

\section{General rights}

Copyright and moral rights for the publications made accessible in the public portal are retained by the authors and/or other copyright owners and it is a condition of accessing publications that users recognise and abide by the legal requirements associated with these rights.

- Users may download and print one copy of any publication from the public portal for the purpose of private study or research.

- You may not further distribute the material or use it for any profit-making activity or commercial gain

- You may freely distribute the URL identifying the publication in the public portal.

If the publication is distributed under the terms of Article 25fa of the Dutch Copyright Act, indicated by the "Taverne" license above, please follow below link for the End User Agreement:

www.tue.nl/taverne

Take down policy

If you believe that this document breaches copyright please contact us at:

openaccess@tue.nl

providing details and we will investigate your claim. 


\title{
Regularization of Moving Boundaries in a Laplacian Field by a Mixed Dirichlet-Neumann Boundary Condition: Exact Results
}

\author{
Bernard Meulenbroek, ${ }^{1}$ Ute Ebert, ${ }^{1,2}$ and Lothar Schäfer ${ }^{3}$ \\ ${ }^{1}$ CWI, P.O. Box 94079, 1090 GB Amsterdam, The Netherlands \\ ${ }^{2}$ Department of Physics, Eindhoven University of Technology, The Netherlands \\ ${ }^{3}$ Department of Physics, Universität Essen, Germany \\ (Received 11 July 2005; published 3 November 2005)
}

\begin{abstract}
The dynamics of ionization fronts that generate a conducting body are in the simplest approximation equivalent to viscous fingering without regularization. Going beyond this approximation, we suggest that ionization fronts can be modeled by a mixed Dirichlet-Neumann boundary condition. We derive exact uniformly propagating solutions of this problem in $2 \mathrm{D}$ and construct a single partial differential equation governing small perturbations of these solutions. For some parameter value, this equation can be solved analytically, which shows rigorously that the uniformly propagating solution is linearly convectively stable and that the asymptotic relaxation is universal and exponential in time.
\end{abstract}

PACS numbers: $52.80 . \mathrm{Mg}, 05.45 .-\mathrm{a}, 41.20 .-\mathrm{q}$

Boundaries between two phases that move according to the gradient of a Laplacian or diffusive field occur in many fields of the natural sciences and have a long and intricate research history [1]; well known examples include viscous fingering in Hele-Shaw flow [2,3], solidification fronts in undercooled melts [1], and migration of steps [4] or electromigration of voids $[5,6]$ on the surface of layered solids or boundaries of bacterial colonies in an external nutrition field [7]. Viscous fingering here takes a paradigmatic role as the oldest and most studied problem-determining the long time dynamics up to today leads to mathematical surprises [8-11].

A similar moving boundary problem arises in so-called streamer discharges $[12,13]$ that precede sparks and lightning. Streamer ionization fronts can be understood as moving boundaries separating an ionized phase from a nonconducting phase [13-15]. The inner front structure can be approximated by a boundary condition of mixed Dirichlet-Neumann type, as we will sketch below. A similar boundary condition appears in step motion on the surface of layered solids when the Schwoebel barrier is taken into account [4]. Our boundary condition has a similar physical effect as the curvature correction in viscous fingering. We show here that it indeed stabilizes certain uniformly translating shapes.

Below we present the following results for the twodimensional version of the problem: (i) Uniformly translating circles are always solutions, though the external electric field breaks the radial symmetry. (ii) Linear perturbations of the translating circles evolve according to a single partial differential equation, which for a certain radius can even be solved analytically. (iii) Planar fronts are linearly unstable to transversal perturbations of arbitrary wave number. Analyzing the stability of the circle within a space restricted to any finite number of $k$ modes, one finds strictly oscillating perturbations. However, our explicit analytical solution for the particular circle radius shows that neither exponentially growing nor strictly oscillating perturbations exist. Rather, after some initial growth all perturbations die out exponentially following a universal trajectory. For $t \rightarrow \infty$ only a shift of the circle remains. Furthermore, while growing, pronounced perturbative structures are convected to the back of the circle, where they disappear. To our knowledge, this is the first explicit solution showing the convective stabilization of a curved front according to the concept of Zel'dovich [16].

In fact, the interfacial dynamics with our boundary condition can be addressed by explicit analysis much further than the classical viscous fingering problem. It therefore might contribute not only to the understanding of ionization fronts but also shed new light on other moving boundary problems such as the classical viscous fingering problem.

A simple moving boundary approximation for a streamer ionization front was suggested by Lozansky and Firsov [17]: The front penetrates into a nonionized and electrically neutral region (indicated with $\mathrm{a}^{+}$) with a velocity determined by the local electric field $\mathbf{E}^{+}=-\nabla \varphi^{+}$:

$$
\nabla^{2} \varphi^{+}=0, \quad v_{n}=\hat{\mathbf{n}} \cdot \nabla \varphi^{+},
$$

where $\hat{\mathbf{n}}$ is the local normal on the boundary. Approximating the interior ionized region as ideally conducting

$$
\varphi^{-}=\text {const, }
$$

and the electric potential as continuous across the ionization boundary $\varphi^{+}=\varphi^{-}$, one arrives at the LozanskyFirsov interfacial model. This model was suggested in Ref. [13] to explain streamer branching, and it was explicitly analyzed in Ref. [15]. Replacing the electric potential $\varphi$ by the pressure field $p$, one finds the nonregularized motion of viscous fingers in a Hele-Shaw cell. The model generically leads within finite time to the formation of 
cusps, i.e., of locations on the interface with vanishing radius of curvature [18].

We here replace the boundary condition $\varphi^{+}=\varphi^{-}$by

$$
\varphi^{+}-\varphi^{-}=\epsilon \hat{\mathbf{n}} \cdot \nabla \varphi^{+}
$$

to suppress these unphysical cusps. Here the length scale $\epsilon$ characterizes the width of the ionization front where the ionization increases and the electric field decreases. It determines the jump $\varphi^{+}-\varphi^{-}$of the electric potential across the boundary for given field $\nabla \varphi^{+}$ahead of the front.

The classical boundary condition for viscous fingering is $\varphi^{+}-\varphi^{-}=-\gamma \kappa$, where $\kappa$ is the local curvature of the moving interface and $\gamma$ is surface tension. In contrast, the boundary condition (3) does not involve front curvature but can be derived from planar ionization fronts, more precisely, from a minimal set of partial differential equations for electron and ion densities and their coupling to the electric field [12]. The formal derivation will be given elsewhere. Here we note that, ignoring electron diffusion $(D=0)$ as in Ref. [14], the planar uniformly translating front solutions of the partial differential equations (PDEs) always yield a relation $\varphi^{+}-\varphi^{-}=F\left(\hat{\mathbf{n}} \cdot \nabla \varphi^{+}\right)$. For large field $E^{+}=\hat{\mathbf{n}} \cdot \nabla \varphi^{+}$ahead of the front, the function $F$ becomes linear, and the boundary condition (3) results.

This boundary condition has a similar physical effect as the curvature correction in viscous fingering: High local fields ahead of the front decrease due to the change of $\varphi^{+}$ on the boundary, and the interface moves slower than an equipotential interface (where $\varphi^{+}=$const). While the boundary condition of viscous fingering suppresses high interfacial curvatures that can lead to high fields, the boundary condition (3) suppresses high fields that frequently are due to high local curvatures. This physical consideration has motivated our present study of whether the boundary condition (3) also regularizes the interfacial motion.

The minimal PDE model for streamer fronts with $D=0$ leads to a dispersion relation with asymptotes

$$
s(k)= \begin{cases}v k & \text { for } k \ll 1 / \epsilon \\ v / \epsilon & \text { for } k \gg 1 / \epsilon\end{cases}
$$

for linear transversal perturbations $e^{i k x+s t}$ of planar interfaces [14]. It is important to check whether the moving boundary approximation (1)-(3) reproduces this behavior. Indeed, analyzing planar interfaces, we find $s(k)=$ $v k /(1+\epsilon k)$ in full agreement with (4) as we will show in detail elsewhere. This strongly suggests that the interfacial model captures the correct physics. It shows, furthermore, that planar fronts are linearly unstable against any wave vector $k$ for all $\epsilon$.

We now restrict the analysis to the two-dimensional version of the model and to arbitrary closed streamer shapes in an electric field that becomes homogeneous

$$
\varphi(x, y) \rightarrow-E_{0} x \quad \text { far from the ionized body. }
$$

The problem is treated with conformal mapping methods
[15]: The exterior of the streamer where $\nabla^{2} \varphi^{+}=0$ can be mapped onto the interior of the unit circle. Parametrizing the original space with $z=x+i y$ and the interior of the unit disk with $\omega$, the position of the streamer can be written as

$$
z=x+i y=f_{t}(\omega)=\frac{1}{h_{t}(\omega)}=\sum_{k=-1}^{\infty} a_{k}(t) \omega^{k},
$$

where $h_{t}(\omega)$ is analytical on the unit disk with a single zero at $\omega=0$ and therefore has the Laurent expansion given on the right. The boundary of the ionized body

$$
\omega=e^{i \alpha}, \quad \alpha \in[0,2 \pi[,
$$

is parametrized by the angle $\alpha$.

The potential $\varphi^{+}$is a harmonic function due to (1); therefore, one can find a complex potential $\Phi(z)=\varphi^{+}+$ $i \psi$ that is analytic. Its asymptote is $\Phi(z) \rightarrow-E_{0} z$ for $|z| \rightarrow \infty$ according to (5). For the complex potential $\hat{\Phi}(\omega)$, this means that

$$
\hat{\Phi}(\omega)=\Phi\left(f_{t}(\omega)\right)=-E_{0} a_{-1}(t)\left(\frac{1}{\omega}+\sum_{k=0}^{\infty} c_{k}(t) \omega^{k}\right),
$$

where the pole $\propto 1 / \omega$ stems from the constant far field $E_{0}$, and the remainder is a Taylor expansion that accounts for the analyticity of $\hat{\Phi}$. The boundary motion $v_{n}=\hat{\mathbf{n}} \cdot \nabla \varphi^{+}$ (1) is rewritten as

$$
\operatorname{Re}\left[i \partial_{\alpha} f_{t}^{*} \partial_{t} f_{t}\right]=\operatorname{Re}\left[-i \partial_{\alpha} \hat{\Phi}\left(e^{i \alpha}\right)\right] .
$$

The boundary condition (3) takes the form

$$
\operatorname{Re}\left[\hat{\Phi}\left(e^{i \alpha}\right)\right]=\epsilon \operatorname{Re}\left[\frac{i \partial_{\alpha} \hat{\Phi}\left(e^{i \alpha}\right)}{\left|\partial_{\alpha} f_{t}\right|}\right] .
$$

Equations (6)-(10) reformulate the moving boundary problem.

For the unregularized problem (where $\epsilon=0$ ), it is well known that all ellipses with a main axis oriented parallel to the external field are uniformly translating solutions: They propagate with velocity $v=-2 E_{0} a_{-1} /\left(a_{-1}-a_{1}\right)$, while the potential is $\hat{\Phi}=E_{0} a_{-1}(t)(\omega-1 / \omega)[15,18]$.

For the regularized problem (where $\epsilon>0$ ), this family of solutions parametrized by both $a_{-1}$ and $a_{1}$ reduces to a one-parameter family, parametrized by $a_{-1}$ only:

$$
\begin{gathered}
z=f_{t}(\omega)=\frac{a_{-1}}{\omega}+v t, \quad \partial_{t} a_{-1}=0, \\
\hat{\Phi}(\omega)=-E_{0} a_{-1}\left(\frac{1}{\omega}-\frac{1-\epsilon / a_{-1}}{1+\epsilon / a_{-1}} \omega\right) .
\end{gathered}
$$

These solutions simply describe circles $z=x+i y=$ $a_{-1} e^{-i \alpha}+v t$ with radii $a_{-1}$ that, according to (9), propagate with velocity $v=-2 E_{0} /\left(1+\epsilon / a_{-1}\right)$. Similar circular solutions in a unidirectional force field have also been found in viscous fingering [19], but there neither 
velocity nor potential depend on the regularization parameter, in contrast to the present problem. Circular viscous fingering solutions in a radially symmetric force field are reported in Ref. [20]. In general, there are only rare cases where nontrivial analytical solutions for a moving boundary problem with regularization can be given [18,21,22].

The physical problem has two length scales, the interface width $\epsilon$ and the circle radius $a_{-1}$. In the sequel, we set $a_{-1}=1$, measuring all lengths relative to the radius of the circle.

Is such a uniformly translating circle stable against small perturbations, in particular, in view of the linear instability of the planar front (4)? The basic equations (6)-(10) show a quite complicated structure, and it is a remarkable feature that linear stability analysis of the translating circle (11) and (12) can be reduced to solving a single partial differential equation. We write

$$
\begin{gathered}
f_{t}(\omega)=\frac{1}{\omega}+\tau+\beta(\omega, \tau), \quad \tau=v t, \quad v=\frac{-2 E_{0}}{1+\epsilon}, \\
\hat{\Phi}(\omega)=-E_{0}\left(\frac{1}{\omega}-\frac{1-\epsilon}{1+\epsilon} \omega\right)+v \phi(\omega, \tau),
\end{gathered}
$$

where $\beta$ and $\phi$ are analytical in $\omega$ and assumed to be small. Equations (9) and (10) are expanded to first order in $\beta$ and $\phi$ about the uniformly translating circle and read

$$
\begin{gathered}
\operatorname{Re}\left[\omega \partial_{\tau} \beta-\omega \partial_{\omega} \beta\right]=\operatorname{Re}\left[-\omega \partial_{\omega} \phi\right] \text { for } \omega=e^{i \alpha,} \\
\frac{\epsilon}{2}\left(\omega+\frac{1}{\omega}\right) \operatorname{Re}\left[\omega^{2} \partial_{\omega} \beta\right]=\operatorname{Re}\left[\epsilon \omega \partial_{\omega} \phi+\phi\right] .
\end{gathered}
$$

By construction, $F(\omega)=\partial_{\tau} \beta-\partial_{\omega} \beta+\partial_{\omega} \phi$ is analytical for $|\omega|<1$, and Eq. (15) shows that $\operatorname{Re}[\omega F(\omega)]=0$ for $|\omega|=1$. Furthermore, it is clear that $\omega F(\omega)$ vanishes for $\omega=0$. Therefore,

$$
0=\omega F(\omega)=\omega\left(\partial_{\tau} \beta-\partial_{\omega} \beta+\partial_{\omega} \phi\right)
$$

is valid on the whole closed unit disk. The corresponding analysis of Eq. (16) yields

$$
\frac{\epsilon}{2}\left(\omega+\frac{1}{\omega}\right) \omega^{2} \partial_{\omega} \beta=\epsilon \omega \partial_{\omega} \phi+\phi+\text { const. }
$$

To this equation the operator $\omega \partial_{\omega}$ is applied, and Eq. (17) is used to eliminate terms containing $\omega \partial_{\omega} \phi$. As a result, we find an equation only for the function $\beta(\omega, \tau)$ :

$$
\begin{gathered}
\mathcal{L}_{\epsilon} \beta=0, \\
\mathcal{L}_{\epsilon}=-\epsilon\left(1-\omega^{2}\right) \omega \partial_{\omega}^{2}-\left(2+\epsilon-3 \epsilon \omega^{2}\right) \partial_{\omega} \\
+2 \epsilon \omega \partial_{\omega} \partial_{\tau}+2(1+\epsilon) \partial_{\tau} .
\end{gathered}
$$

Equation (19) has to be solved for arbitrary initial conditions $\beta(\omega, 0)$ that are analytical in some neighborhood of the unit disk. The operator $\mathcal{L}_{\epsilon}$ conserves analyticity in time. $\epsilon$ is a singular perturbation that multiplies the highest derivatives $\partial_{\omega}^{2}$ and $\partial_{\omega} \partial_{\tau}$.
The case $\epsilon=0$ is almost trivial, since $\mathcal{L}_{\epsilon}$ reduces to

$$
\mathcal{L}_{0}=2\left(\partial_{\tau}-\partial_{\omega}\right) .
$$

Thus, all solutions can be written as

$$
\beta(\omega, \tau)=\hat{\beta}(\omega+\tau),
$$

where $\hat{\beta}(\zeta)$ is any function analytic in a neighborhood of the unit disk $|\zeta| \leq 1$. The time evolution just amounts to a translation along the strip $-1 \leq \operatorname{Re} \zeta \leq \infty,|\operatorname{Im} \zeta| \leq 1$. Any singularity of $\hat{\beta}$ at some finite point $\zeta$ on the strip will lead to a breakdown of perturbation theory within finite time; this is the generic behavior as found previously in the full nonlinear analysis of this unregularized problem. Of course, there also exist solutions that stay bounded for all times.

A different perspective on $\epsilon=0$ is that the Richardson moments are an infinite sequence of conserved quantities [18]. A reflection of this property is that any polynomial $\beta(\omega, \tau)=\sum_{k=0}^{N} b_{k}(\tau) \omega^{k}$ for any $N$ with an appropriate choice of the time dependent functions $b_{k}(\tau)$ is an exact solution for all times $\tau>0$, even for the full nonlinear problem [15]. This suggests that an expansion in powers of $\omega$ is a natural ansatz also for nonvanishing $\epsilon$. Taking as initial condition some polynomial of order $N$, one finds from the form (20) of $\mathcal{L}_{\epsilon}$ that higher modes $\omega^{k}, k>N$ are generated dynamically - similarly to the daughter singularities in regularized viscous fingers [8]. When the expansion in $\omega$ is truncated at some arbitrary $N^{\prime}$, it can be shown that the problem for any truncation $N^{\prime}$ and for any value $\epsilon>0$ has purely imaginary temporal eigenvalues. One would, therefore, expect all eigensolutions for $\epsilon>0$ to be purely oscillating in time. However, this behavior disagrees with our exact solution for $\epsilon=1$.

For $\epsilon=1$, it turns out that the operator factorizes

$$
\mathcal{L}_{1}=\left[2 \partial_{\tau}-\left(1-\omega^{2}\right) \partial_{\omega}\right]\left[2+\omega \partial_{\omega}\right],
$$

which allows us to construct the general solution $\beta(\omega, \tau)$ of (19). We introduce the function

$$
g(\omega, \tau)=\left[2+\omega \partial_{\omega}\right] \beta(\omega, \tau)
$$

that obeys the equation

$$
\left[2 \partial_{\tau}-\left(1-\omega^{2}\right) \partial_{\omega}\right] g(\omega, \tau)=0 .
$$

The general solution of this equation reads

$$
g(\omega, \tau)=G\left(\frac{\omega+T}{1+T \omega}\right), \quad T=\tanh \frac{\tau}{2} .
$$

The function $G$ is derived from the initial condition as

$$
G(\omega)=g(\omega, 0)=\left[2+\omega \partial_{\omega}\right] \beta(\omega, 0) ;
$$

hence, it is analytical in a neighborhood of the unit disk. Finally, Eq. (24) is solved by

$$
\beta(\omega, \tau)=\int_{0}^{\omega} \frac{x d x}{\omega^{2}} G\left(\frac{x+T}{1+T x}\right) .
$$




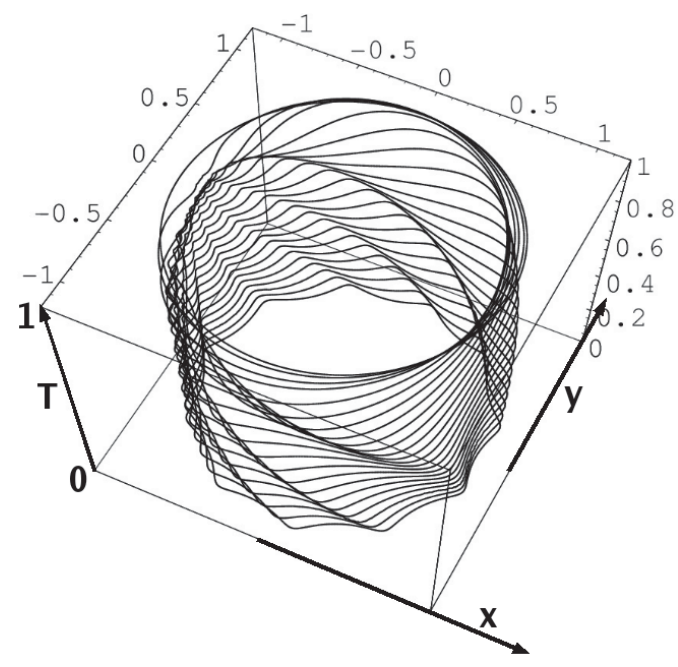

FIG. 1. Temporal evolution of a perturbed circle $f_{t}(\omega)-\tau=$ $1 / \omega+\beta(\omega, \tau)$ moving in the positive $x$ direction, according to Eqs. (12) and (26)-(28). The uniform translation $\tau$ is subtracted. The initial perturbation is a Fourier mode $\beta(\omega, 0)=-0.5 \omega^{k} /(k+2)$ with $k=10$. The evolution during times $0 \leq \tau \leq \infty$ corresponding to $0 \leq T \leq 1$ is shown for time steps $T=0,0.05,0.1, \ldots, 0.85,0.9,0.95,0.97,0.99,1.0$.

Now the one-parameter family of mappings

$$
\omega \rightarrow \zeta_{T}(\omega)=\frac{\omega+T}{1+T \omega}, \quad-1<T<1,
$$

forms a subgroup of the automorphisms of the unit disk. Thus, on the level of $G(\zeta)$, the dynamics amounts to a conformal mapping of the unit disk $|\omega| \leq 1$ onto itself. This dynamics is somewhat distorted by the additional integration (28) leading to $\beta(\omega, \tau)$, but it is easily seen that $\beta(\omega, \tau)$ and $\partial_{\omega} \beta(\omega, \tau)$ are bounded uniformly in $\tau$ for $|\omega| \leq 1$. Hence, contrary to the unregularized problem for $\epsilon=0$, only perturbations contribute that are bounded for all times. Hence, an infinitesimal perturbation can never form cusps. Furthermore, the mapping $\omega \rightarrow \zeta_{T}(\omega)$ has fixed points $\omega= \pm 1$; and for $\tau \rightarrow \infty$, i.e., $T \rightarrow 1$, it degenerates to $\zeta_{1}(\omega) \equiv 1$, provided $\omega \neq-1$. Expanding (28) about $T=1$, we thus find the asymptotic behavior

$$
\begin{aligned}
\beta(\omega, \tau) \stackrel{\tau \rightarrow \infty}{\rightarrow} & \frac{G(1)}{2}+G^{\prime}(1)\left[1-\frac{4}{\omega}+\frac{4 \ln (1+\omega)}{\omega^{2}}\right] e^{-\tau} \\
& +O\left(e^{-2 \tau}\right) \quad \text { for } \omega \neq-1 .
\end{aligned}
$$

(For $\omega=-1$, relaxation is proportional to $\tau e^{-\tau}$.) Here the initial conditions determine only the complex numbers $G(1)$ and $G^{\prime}(1)$ in an otherwise universal asymptotic relaxation. For $\tau \rightarrow \infty$, the perturbation just shifts the basic circular solution without change of shape, and the relaxation is exponential in time and follows a universal slow manifold. Indeed, it is easily checked that any pronounced structure of the initial perturbation that is not located right at the top at $\omega=1$ is convected with increasing time toward $\omega=-1$, where it vanishes according to (30). This initial convective stabilization is an outflow of the simple dynamics of $G(\zeta)$ as pointed out above. Figure 1 illustrates this behavior.

To summarize, we have found that the boundary condition (3) at least for $\epsilon=1$ regularizes our problem in the sense that an infinitesimal perturbation of a uniformly translating circle stays infinitesimal for all times and vanishes asymptotically for $\tau \rightarrow \infty$ up to an infinitesimal shift of the complete circle. This statement is based on an exact analytical solution for an arbitrary initial perturbation. At the present stage, we have indications that this behavior of infinitesimal perturbations might be generic for $\epsilon>0$, while the solution is unstable for $\epsilon=0$. Furthermore, we expect that the convection of perturbations to the back of the structure applies similarly for other shapes such as fingers. When applying the present calculation to streamers, we in fact have to assume this to be true, since streamers are typically not closed bodies but, rather, the tips of ionized channels. Finally, the behavior of finite perturbations and their nonlinear analysis will require future investigations.

[1] P. Pelcé, Dynamics of Curved Fronts (Academic, Boston, 1988).

[2] P. G. Saffman and G. I. Taylor, Proc. R. Soc. A 245, 312 (1958).

[3] D. Bensimon, L. P. Kadanoff, S. Liang, B. I. Shraiman, and C. Tang, Rev. Mod. Phys. 58, 977 (1986).

[4] P. Politi et al., Phys. Rep. 324, 271 (2000).

[5] M. Ben Amar, Physica (Amsterdam) 134D, 275 (1999).

[6] P. Kuhn, J. Krug, F. Hausser, and A. Voigt, Phys. Rev. Lett. 94, 166105 (2005).

[7] J. Müller and W. van Saarloos, Phys. Rev. E 65, 061111 (2002).

[8] M. Siegel and S. Tanveer, Phys. Rev. Lett. 76, 419 (1996).

[9] S. Tanveer, J. Fluid Mech. 409, 273 (2000).

[10] D. A. Kessler and H. Levine, Phys. Rev. Lett. 86, 4532 (2001).

[11] J. Casademunt, Chaos 14, 809 (2004).

[12] U. Ebert, W. van Saarloos, and C. Caroli, Phys. Rev. E 55, 1530 (1997).

[13] M. Arrayás, U. Ebert, and W. Hundsdorfer, Phys. Rev. Lett. 88, 174502 (2002).

[14] M. Arrayás and U. Ebert, Phys. Rev. E 69, 036214 (2004).

[15] B. Meulenbroek, A. Rocco, and U. Ebert, Phys. Rev. E 69, 067402 (2004).

[16] Ya. B. Zel'dovich, A. G. Istratov, N. I. Kidin, and V.B. Librovitch, Combust. Sci. Technol. 24, 1 (1980).

[17] E. D. Lozansky and O. B. Firsov, J. Phys. D: Appl. Phys. 6, 976 (1973).

[18] V. M. Entov, P. I. Etingof, and D. Ya. Kleinbock, Eur. J. Appl. Math. 4, 97 (1993).

[19] S. Tanveer, Phys. Fluids 29, 3537 (1986).

[20] R. Folch et al., physics/0408092.

[21] G.L. Vasconcelos and L.P. Kadanoff, Phys. Rev. A 44, 6490 (1991).

[22] D. Crowdy, J. Fluid Mech. 409, 223 (2000). 